\title{
Drag force in asymptotically Lifshitz spacetimes
}

\author{
K Bitaghsir Fadafan \\ Department of Physics, Shahrood University of Technology, Shahrood, Iran \\ E-mail: bitaghsir@shahroodut.ac.ir
}

(Received 10 March 2018 ; in final form 17 August 2019)

\begin{abstract}
We calculated the drag force for asymptotically Lifshitz space times in $(d+2)$-dimensions with the arbitrary dynamical exponent $z$. We find that at zero and finite temperature, the drag force has a non-zero value. Using the drag force calculations, we investigate the DC conductivity of the strange metals.
\end{abstract}

Keywords: AdS/CFT correspondence, string theory, drag force, conductivity, strange metals

\section{Introduction}

A new method for studying different aspects of strongly coupled quantum field theories is the AdS/CFT correspondence [1-4], which has yielded many important insights into the dynamics of strongly coupled field theories. Recently, the application of this duality in condensed matter physics, called AdS/CMT, has been studied [5]. This duality is very useful to study certain strongly coupled systems in CMT by holography techniques and to better understand their properties.

Methods based on AdS/CFT relate gravity in the $\mathrm{AdS}_{d+2}$ space to the conformal field theory on the $(d+1)$-dimensional boundary. These conformal field theories are invariant under the following scaling transformation

$$
(t, \vec{x}) \rightarrow(\lambda t, \lambda \vec{x})
$$

However, in many condensed matter systems, there are field theories with anisotropic scaling symmetry. This unconventional scaling can be illustrated as

$$
(t, \vec{x}) \rightarrow\left(\lambda^{\mathrm{z}} t, \lambda \vec{x}\right)
$$

where $\mathrm{z}$ is the dynamical exponent. These field theories exist near a critical phenomenon and describe multicritical points in certain magnetic materials and liquid crystals. In the case of $\mathrm{z}=1$, theory benefits from the relativistic scale invariance. For $\mathrm{z}=2$, there is a $2+1$ dimensional field theory so-called as Lifshitz field theory. This theory has a line of fixed points parameterized by $\kappa$ and the lagrangian density, which is given by

$$
\mathcal{L}=\int d x^{2} d t\left(\left(\partial_{t} \phi\right)^{2}-\kappa\left(\nabla^{2} \phi\right)^{2}\right)
$$

These fixed points are strongly coupled, appearing in strongly correlated electrons in zero and finite temperature lattice models [8]. Another important theory with unconventional scaling in (2) is the theory whose symmetry group is the Schrodinger group $\operatorname{Sch}(d-1)$, and the geometry is given by a deformation of the AdS geometry [6]. Many of the most interesting examples in the condensed matter theory arise in the Lifshitz case.

In this paper, we consider massive charge carriers described by the flavor branes in the Lifshitz space times. As discussed in [9], considering the massive charge carriers in this background is the case of interest in the modeling of strange metals. Gravity duals have been investigated in [7].

To calculate the drag force, one should consider a probe brane in this background. Adding flavor branes and finitedensity holography has been studied in $[11,12]$. These probe branes are related to a quantum critical theory and we consider massive charge carriers interacting with this theory. Notice that these carriers do not backreact on the quantum critical system. In the massive case, the flavor brane forms a cigar-like shape with its tip at $r_{0}$ and the charge carriers correspond to the strings stretching from the tip of the cigar down to the horizon. At finite temperature, one should consider a black brane in the background at a finite radial position, $r_{h}$. As a result, charge carriers on the flavor brane correspond to the stretching strings from $r_{0}$ to the horizon. To calculate the drag force, one should consider a stretching string from the flavor brane and use the prescription in $[16,17,18]$ to find the energy loss at zero and finite temperature cases.

The most useful application of drag calculations in the Lifshitz background has been done in [9]. They have studied the phenomenology of strange metals and computed the electrical conductivity. We discuss this application of the drag force in the last section. Using the drag force calculations, we 
investigate the DC conductivity of strange metals and derive some results [9].

This paper is organized as follows. In the next section, we use the proposed solutions in [22-24] and discuss the energy loss of the massive charge carriers. We find that they lose energy even at the zero temperature. We compare the result obtained for the energy loss of the particles computed in the case of the non-relativistic gravity dual to the field theory based on the Schrodinger CFT symmetry [13]. Also, in this case, the moving particle loses energy at the zero temperature. In the section three, we consider the Lifshitz background embedded into the string theory [38]. We also find a non-zero drag force at the zero temperature. In the last section, we discuss computing the DC conductivity from drag calculations. While this paper was in the final stages of preparation, it came to our attention that the drag force in non-relativistic background whose symmetry is Schrodinger group has been done in [39]. This calculation extends the results of [13] and confirms a non-zero drag force at the zero temperature.

\section{Asymptotic Lifshitz space times}

In this section, we provide the backgrounds necessary for our discussions. We consider the non-relativistic holography in [22]. In this study, Lifshitz geometry is a solution of the gravity coupled to a massive vector field. The $(\mathrm{d}+2)$-dimensional spacetime action is

$$
\begin{aligned}
S= & \frac{1}{16 \pi G_{d+2}} \int d^{d+2} x \sqrt{-g} \\
& {\left[R-2 \Lambda-\frac{1}{2} \partial_{\mu} \phi \partial^{\mu} \phi-\frac{1}{4} e^{\lambda \phi} F_{\mu v} F^{\mu v}\right] . }
\end{aligned}
$$

where $\Lambda$ is the cosmological constant and massless scalar field and abelian gauge field are the matter fields of theory. The only non-vanishing components of the field strength is $F_{r t}=q e^{-\lambda \phi} r^{Z-d-1}$, and $q$ is related to the charge of the black hole. Based on this action, one finds the asymptotically Lifshitz solution at the zero temperature

$$
\begin{aligned}
& d s^{2}=L^{2}\left(-r^{2 z} d t^{2}+\frac{d r^{2}}{r^{2}}+r^{2} \sum_{i=1}^{d} d x_{i}^{2}\right), \\
& F_{r t}=q e^{-\lambda \phi} r^{z-d-1}, e^{\lambda \phi}=r^{\lambda \sqrt{2(z-1)^{d}}}, \\
& \lambda^{2}=\frac{2 d}{z-1}, q^{2}=2 L^{2}(z-1)(z+d), \\
& \Lambda=-\frac{(z+d-1)(z+1)}{2 L^{2}} .
\end{aligned}
$$

In this solution, dilaton is not constant. However, exact solutions can be found. To calculate the drag force, one should consider the flavor probe branes in the background (5) and study the moving massive charge carriers. From gauge-string duality, we consider a trailing open string in the holographic direction. The action of this open string is given by the NambuGoto action

$$
S=-T_{0} \int d \tau d \sigma \sqrt{-g} .
$$

where $T_{0}$ is the tension of the string. The coordinates $(\sigma, \tau)$ parameterize the induced metric $g_{a b}$ on the string world-sheet and $g$ is the determinant of the world-sheet metric $g_{a b}$,

$$
-g=-\operatorname{detg}_{a b}=\left(\dot{X} \cdot X^{\prime}\right)^{2}-\left(X^{\prime}\right)^{2}(\dot{X})^{2},
$$

where $X^{\mu}(\sigma, \tau)$ is a map from the string world-sheet into space-time, and we define $\dot{X}=\partial_{\tau} X, X^{\prime}=\partial_{\sigma} X$, and
$V . W=V^{\mu} W^{v} G_{\mu v}$, where $G_{\mu v}$ is the metric. The Lagrangian density is given by $\mathcal{L}=-T_{0} \sqrt{-g}$. The string equation of motion is obtained as

$\partial_{\rho}\left(\frac{\partial \mathcal{L}}{\partial \dot{x}}\right)+\partial_{t}\left(\frac{\partial \mathcal{L}}{\partial \dot{x}}\right)=0$.

One has to calculate the canonical momentum densities, $\pi_{x}^{0}, \pi_{t}^{0}$, to find the total energy and momentum of the moving particle in non-relativistic field theory

$E=\int_{\rho h}^{\rho 0} d \rho \pi_{t}^{0}, P=-\int_{\rho h}^{\rho 0} d \rho \pi_{x}^{0}$.

\subsection{Drag force at the zero temperature}

Now, we calculate the drag force at the zero temperature. We consider a moving heavy point particle on the probe flavor brane in the d-dimensional space with the following ansatz

$t=\tau, r=\sigma, x_{1}=x=v t+\xi(r), x_{i}=0(i \neq 1)$,

One finds from the equations of motion (2.5) that

$\dot{\xi}^{2}=\frac{C^{2}\left(r^{2 z-2}-v^{2}\right)}{r^{2 z+2}\left(r^{2 z+2}-C^{2}\right)}$,

where $\mathrm{C}$ is the constant of motion. The drag force experienced by the moving particle is

$F_{\text {drag }}=-T_{0} v^{2\left(\frac{z+1}{z-1}\right)}$.

The drag force is independent of the dimension of space. In (10), we considered the string from boundary to infinity; then one finds from the numerator that there is no bound on the velocity and it can change from zero to infinity. This is because of the fact that the dual theory is non-relativistic. This is an interesting result because even though the system is at zero temperature, the moving particle loses its energy. We consider the mass and momentum of the particle as $\mathrm{M}$ and $\mathrm{P}$, respectively. Then $P=M v$; in the case of constant momentum, the drag force will be found as $F_{\text {drag }}=\mu M v$. In this way, one finds the friction term as $\mu=\left(T_{0} / M\right) v^{[(z+3) / z-1]}$.

Authors in ref. [13] found a non-zero drag force at the zero temperature. They studied the non-relativistic three dimensional CFT at the zero and finite temperature. They found that unlike the AdS case, where one only gets a casual speed limit, in the non-relativistic case, one arrives at no speed limit and the non-zero drag force. We also find the same results in asymptotically Lifshitz spacetimes with the arbitrary critical exponent. As a result, the non-zero drag force could be considered as a common property of non-relativistic spacetimes.

\section{2. Drag force at finite temperature}

Unlike the case of the Schrödinger conformal group, it is difficult to obtain analytic black hole solutions in Lifshitz spacetimes. In fact, the problem of finding analytic exact black hole solutions with asymptotically Lifshitz geometry has turned out to be a highly non-trivial problem. However, there are known solutions. For example, black hole solutions with $z=2$ in four dimensions were studied in [15] and black holes in asymptotically Lifshitz spacetimes with the arbitrary critical exponent were investigated in [20]. Topological black holes and other solutions were proposed in [21, 22]. For other recent solutions on Lifshitz black holes, see [26-32]. We consider the black brane solution of (4) in asymptotic Lifshitz (2+1)- 
dimensional spacetime, as proposed in [22]

$$
\begin{aligned}
& d S^{2}=L^{2}\left(-r^{2 z} f(r) d t^{2}+\frac{d r^{2}}{r^{2} f(r)}+r^{2} d \vec{x}_{i}^{2}\right), \\
& f(r)=1-\left(\frac{r_{+}}{r}\right)^{z+d} .
\end{aligned}
$$

The matter fields are a massless scalar and an abelian gauge field and the other fields remain the same as those in the zero temperature case. The temperature is

$T_{H}=\frac{(z+d) r_{+}^{z}}{4 \pi}$,

and the black hole entropy is given by

$S_{B}=\frac{V_{d} L^{d} r_{+}^{d}}{4 G_{d+2}}$,

where $\mathrm{V}_{d}$ is the volume of the $\mathrm{d}$ dimensional spatial coordinates $\overrightarrow{x_{i}}$.

We consider the moving heavy point particle in the $x$ direction and by following the ansatz in (10), one finds that

$$
\dot{\xi}^{2}=\frac{C^{2}\left(r^{2 z-2}-\frac{v^{2}}{f(r)}\right)}{f(r) r^{2 z+2}\left(L^{4} f(r) r^{2 z+2}-C^{2}\right)},
$$

where $\mathrm{C}$ is the constant of motion. From the reality condition of the above equation, one finds that $C=v L^{2} r_{C}^{2}$ and $r_{C}$ is the root of this equation

$$
\left(1-\left(\frac{r_{+}}{r_{c}}\right)^{z+d}\right) r_{c}^{2 z-2}-v^{2}=0,
$$

The drag force is given by

$$
F_{\text {drag }}=-T_{0} v L^{2} r_{c}^{2} \text {. }
$$

We consider some special cases and derive the drag force as follows.

In the case $d=2$ and $z=1$, there are two dimensional spatial coordinates and $z=1$ means the isotropic theory. The drag force in terms of the temperature of the field theory, $T_{H}=3 r_{+} / 4 \pi$, is given by

$$
F_{\text {drag }}=-T_{0} L^{2}\left(\frac{4 \pi}{3}\right)^{\frac{2}{3}} T_{H}^{\frac{2}{3}} \frac{v}{\left(1-v^{2}\right)^{\frac{2}{3}}} .
$$

Another specific example is known as the Lifshitz model. For $d=2$ and $z=2$ this case appears in the systems of strongly correlated electrons in condensed matter physics. Having a holographic description for these phenomena would be of great importance to investigate some properties of strongly coupled systems in the condensed matter. One finds the drag force as

$$
F_{\text {drag }}=-T_{0} L^{2} v^{2}\left(\frac{v^{2}}{2}-\sqrt{\frac{v^{2}}{2}+\pi^{2} T_{H}^{2}}\right) .
$$

It is clear that in each case, the temperature dependency of the drag force is different. It is straightforward to discuss the drag force in other cases with spatial dimensions more than $d=2$ and different values for $z$.

\section{3. $R^{2}$ corrections of the drag force}

Now, we study $R^{2}$ corrections. These corrections for fivedimensional asymptotically Lifshitz spacetimes have been studied in [24]. The specific example is the Gauss- Bonnet model. Black brane solution has been found perturbatively as

$$
\begin{aligned}
d S^{2}=L^{2}\left[-g(u)(1-u) d t^{2}\right. & +\frac{1}{h(u)(1-u)} d u^{2} \\
& +\frac{r_{+}^{2}}{u^{A}}\left(d x_{1}^{2}+d x_{2}^{2}+d x_{3}^{2}\right],
\end{aligned}
$$

where

$$
\begin{array}{r}
g(u)=r_{+}^{2 z} u^{-\frac{4 z}{z_{0}+3}}(1+u)\left(1+\lambda_{G B}\left(1-u^{2}\right)\right) \\
\exp \left[4 \lambda_{G B} \frac{z_{0}-1}{z_{0}+3} u^{2}\right], \\
h(u)=\frac{1}{4}\left(z_{0}+3\right)^{2} u^{2}(1+u)\left(1+\lambda_{G B}\left(1-u^{2}\right)\right) .
\end{array}
$$

in which $\lambda_{G B}$ is the Gauss-Bonnet coupling constant. And

$$
u^{2}=\left(\frac{r_{+}}{r}\right)^{z_{0}+3} A=\frac{4}{z_{0}+3}, \quad z=z_{0}+2 \lambda_{G B}\left(z_{0}-1\right) \text {. }
$$

The horizon of the black brane is located at $u=1$ and the boundary is at $u=0$. The Hawking temperature is

$$
T_{H}=\frac{\left(z_{0}+3\right) r_{+}^{z}}{4 \pi}\left(1+2 \lambda_{G B}\left(\frac{z_{0}-1}{z_{0}+3}\right)\right) \text {. }
$$

The drag force in the case of $z_{0}=1$ has been calculated in [25].

We consider the moving heavy point particle in the $\$ x \$$ direction and by following the ansatz in (10), one finds that

$$
\dot{\xi}^{2}=\frac{C^{2}\left(\frac{g(u)}{h(u)}-\frac{r_{+}^{2} v^{2}}{u^{A} h(u)(1-u)}\right)}{\frac{r_{+}^{2}(1-u) g(u)}{u^{A}}\left(\frac{L^{4} r_{+}^{2}(1-u) g(u)}{u^{A}}-C^{2}\right)},
$$

From the reality condition of the above equation, we should find the roots of this equation

$g\left(u_{c}\right)-\frac{r_{+}^{2} v^{2}}{u_{c}^{A}\left(1-u_{c}\right)}=0$,

Regarding this equation, the drag force can be found as

$$
F_{\text {drag }}=-T_{0} r_{+}^{2} v\left(\frac{1+u_{c}}{u_{c}^{A}}\right) \text {. }
$$

Notice that the dependency of the drag force on the temperature of the field theory is complicated.

One can also study the charge effects on the drag force. The charged Lifshitz black hole solutions in the general $(d+2)$ - dimensions have been investigated in [25]. Also, the Gauss-Bonnet corrections to such black holes in five dimensions have been calculated perturbatively. Using these solutions, one can study the drag force in these backgrounds.

\section{Drag force in the string duals of non-relativistic Lifshitz-like theories}

The aim of this section is to study some features of $(1+2)$ dimensional non-relativistic field theory using the supergravity solution in the type IIB string theory. Since we are dealing with string theory, it is natural to consider a semi-classical string in this background. However, it is difficult to embed the Lifshitz background into the string theory. Some no-go theorems for the string theory duals of non-relativistic Lifshitz-like theories have been proposed in [36]. They propose that classical solutions in type IIA and eleven-dimensional supergravities are not possible. (These solutions are expected to be dual to $(2+1)$ - 
dimensional Lifshitz-like theories.). Based on the holographic constructions of the fractional quantum Hall effect (FQHE) via string theory, authors of [37] have proposed D3-D7 solutions. Using this construction, the embedding of the anisotropic background into type IIB string theory was studied in [38]. However, the scaling behavior in this solution is different and the anisotropy of the scale transformation is only through one of the three spatial directions. As a result, it corresponds to a classical Lifshitz point. Also, since it has a non-constant dilaton, the anisotropic scale invariance only holds at the leading order of interactions. In the context of AdS/CFT correspondence, an open string can be associated to a Wilson loop in the dual field theory and one can consider particle at the end of this semi-classical open string at the boundary. Regarding this study, we consider a moving point particle in a strongly correlated system and calculate the drag force.

\subsection{Drag force at the zero temperature}

We study the drag force at the zero temperature. Spacetime metric in the Einstein frame is given by [38]

$$
d s_{E}^{2}=\tilde{R}^{2}\left[r^{2}\left(-d t^{2}+d x^{2}+d y^{2}\right)+r^{\frac{4}{3}} d w^{2}+\frac{d r^{2}}{r^{2}}\right]+R^{2} d s_{X_{5}}^{2},
$$

This metric is invariant under the scaling

$$
(t, x, y, w, r) \rightarrow\left(\lambda t, \lambda x, \lambda y, \lambda^{\frac{2}{3}} w, \frac{r}{\lambda}\right)
$$

; therefore, it is expected to be holographically dual to Lifshitzlike fixed points with space-like anisotropic scale invariance. One can redefine the radius coordinate $\rho \equiv r^{2 / 3}$ and rescale $(t, x, y, w)$ accordingly. Then the metric in the string frame will be as follows:

$$
d s_{E}^{2}=\tilde{R}^{2}\left[\rho^{3}\left(-d t^{2}+d x^{2}+d y^{2}\right)+\rho^{2} d w^{2}+\frac{d \rho^{2}}{\rho^{2}}\right]+R^{2} d s_{X_{5}}^{2} .
$$

This can be regarded as the gravity duals of Lifshitz-like fixed points with $z=3 / 2$.

Now, we study a moving heavy particle in the "x" and "w" directions. We expect different behaviors in two directions. This is because the "w" direction is an anisotropic direction, but " $\mathrm{x}$ " is not.

\subsubsection{Moving in the $x$ direction, a time dependent solution}

In this case, we study the moving particle in the $x$ direction and consider a time-dependent solution as the following ansatz

$$
X^{\mu}=(t=\tau, x=v t+\xi(\rho), y=0, w=0, \rho)
$$

one finds the Nambu-Goto action

$$
S=-\frac{1}{2 \pi \alpha^{\prime}} \int d t d_{\rho} \tilde{R}^{2} \sqrt{\rho\left(1-v^{2}\right)+\xi^{\prime} 2 \rho^{6}} .
$$

It would be straightforward to calculate $\xi^{\prime}$ from the above equation

$$
\xi^{\prime 2}=\frac{A^{2} \rho\left(1-v^{2}\right)}{\rho^{6}\left(\tilde{R}^{4} \rho^{6}-A^{2}\right)},
$$

where $\mathrm{A}$ is the constant of motion. By studying the realitycondition for the lagrangian density $\mathcal{L}=-T_{0} \sqrt{-g}$ and therefore, for $\xi^{\prime 2}$, one finds that the constant of motion can be chosen arbitrary. In the special case of moving with the speed of light, one finds $F_{\text {drag }}=-T_{0} \tilde{R}^{2} \rho^{3}$. One observes that there is a bound on the velocity which was expected because the $x$ direction is not the anisotropic direction.

\subsubsection{Moving in the $\omega$ direction, a time dependent solution}

In this case, we ask about the moving non-relativistic particle in the anisotropic direction. The ansatz is $\omega=v t+\xi(\rho)$ and from the Nambu-Goto action (6), one finds that

$$
\xi^{2}=\frac{B^{2}\left(\rho-v^{2}\right)}{\rho^{5}\left(\rho^{5}-B^{2}\right)}
$$

$\xi^{\prime 2}$ must be a real parameter and with this condition, $B$ can be found. Also, it is clear from the numerator of (34) that there is no bound on the velocity of particle and it can be changed from zero to infinity. This result is reasonable, because the "w" direction is anisotropic and the dual theory is non-relativistic. As a result, one finds a non-zero drag force on a moving particle in the $w$ direction and at the zero temperature, as represented

$$
F_{\text {drag }}=\frac{d p}{d t}=-T_{0} B=-T_{0} v^{5} .
$$

One can consider the momentum and mass of particle as $P$ and $M$, respectively, and use the non-relativistic relation $P=M v$. We rewrite the drag force in terms of $P$ and derive the momentum of particle as

$$
p(t)=\left(\frac{4 T_{0}}{M^{5}}\right)^{\frac{1}{4}} \frac{1}{t^{\frac{1}{4}}} \text {. }
$$

It would be interesting to calculate the friction coefficient of the moving particle. Using the relation $\dot{P}=-\mu M v$, one finds that the friction coefficient is velocity dependent, $\mu=\frac{T_{0}}{M} v^{4}$.

In this case, although the system is at the zero temperature, the moving particle loses its energy. This phenomena has also been found by [13], where they considered the non-relativistic three dimensional CFT at the zero temperature.

\subsection{Drag force at finite temperature}

It was shown that an $A d S$ space with a black brane is dual to the conformal field theory at the finite temperature [4]. We use the extension of the AdS/CFT correspondence in the case of an anisotropic spacetime. This gravity dual is known as the string theory duals Lifshitz-like fixed points [38]. The metric in the Einstein frame is

$$
\begin{array}{r}
d s_{E}^{2}=\tilde{R}^{2}\left[r^{2}\left(-f(r) d t^{2}+d x^{2}+d y^{2}\right)+r^{\frac{3}{4}} d w^{2}\right. \\
\left.+\frac{d r^{2}}{r^{2} f(r)}\right]+R^{2} d s_{X_{5}}^{2},
\end{array}
$$

where

$$
f(r)=1-\frac{\mu}{r^{11 / 3}} \text {. }
$$

The constant $\mu$ represents the mass parameter of the black brane. The Hawking temperature is

$T_{H}=\frac{11}{12 \pi} \mu^{11 / 3}$.

We study a moving object in the hot gauge theory and in the bulk space, a moving open string should be considered too. Based on the Nambu-Goto action of string in (6) and the equation of motion in (8), the simplest solution for the equation of motion is 
$x=$ constant. In this case, the string is stretched from the probe D-brane at $\rho=\rho_{m}$ to the horizon at $\rho=\rho_{h}$, straightforwardly. In other words, we have a static particle without any motion. The energy of particle in this case is associated with the rest mass of the particle, which is obtained by

$M_{\text {rest }}=\frac{T_{0} \tilde{R}^{2}}{2}\left(\rho_{m}^{2}-\rho_{h}^{2}\right)$.

One can compare this result with the rest mass of a static quark in the $\mathcal{N}=4 S Y M$ theory [16]. In our study, one may use the relation $E_{\text {gap }}=M C^{2}$ and interpret (40) as the energy scale of bulk excitations at the position of the flavor brane.

\subsection{Moving in the $x$ direction, a time dependent solution}

We consider the moving particle in the $x$ direction and consider a time-dependent ansatz $x=v t+\xi(r)$; with this choice, one finds that

$$
\dot{\xi}^{2}=\frac{1-C^{2} \frac{v^{2}}{f(r)}}{r^{4} f(r)\left(\tilde{R}^{4} r^{4} f(r)-C^{2}\right)},
$$

From this equation, we can find the critical radius where numerator and denominator change their sign

$$
r_{c}=\left(\frac{\mu}{1-v^{2}}\right)^{\frac{3}{11}} \text {, }
$$

Finally, the drag force on the particle is given by

$$
\frac{d p}{d t}=-\left(\frac{12 \pi}{11}\right)^{2} \tilde{R}^{2} T_{H}^{2} \frac{v}{\left(1-v^{2}\right)^{\frac{6}{11}}} \text {. }
$$

It is clear that the moving particle loses its energy at the finite temperature case too.

\subsection{Moving in the $w$ direction, a time-dependent solution}

It would be interesting to study a moving object in the "w" direction. This is an anisotropic direction in which the spacetime violates the Lorentz symmetry. One can consider the following ansatz

$$
X^{\mu}=(t=\tau, x=0, y=0, w=v t+\xi(r), r) .
$$

The constant of motion can be considered as $D$ and from the equation of motion, one finds that

$$
\dot{\xi}^{2}=\frac{D^{2}\left(1-\frac{v^{2} r^{\frac{-2}{3}}}{f(r)}\right)}{r^{\frac{10}{3}} f(r)\left(\tilde{R}^{4} r^{\frac{10}{3}} f(r)-D^{2}\right)},
$$

Both numerator and denominator must change sign at the same root; from the numerator, one should solve $r^{11 / 3}-\mathrm{v}^{2} r^{3}-\mu=0$ to find the drag force. We name the root as $r_{\mathrm{c}}$ and find the drag force as

$$
F_{\text {drag }}=-T_{0} v r_{c}^{4 / 3} \text {. }
$$

Based on this relation, one can discuss the drag force too.

\section{Discussion}

In this paper we have calculated the drag force for asymptotically Lifshitz space times in the $(d+2)$ - dimensions with the arbitrary dynamical exponent $z$ from gauge-string duality. We have used the proposed solutions in [22- 24]. The finite temperature behavior of Wilson loops as an application to strongly coupled gauge theories in $3+1$ dimensions has been studied in $[14,15$,$] . By analyzing action in (1.3), one concludes$ that the boundary theory can be viewed as a gauge theory in $2+1$ dimensions with a dimensionless coupling constant; as a result, the theory may have some features in common with the conventional gauge theory in $3+1$ dimensions [15]. Having the Wilson loops on the gravity side, one can study the drag force in the gauge theory side. From gauge-string duality, one should consider a hanging string from the boundary to the horizon. The end point of the string represents the particle that is charged under the gauge field. We have considered a moving heavy point particle and calculated the drag force at zero and finite temperature non-relativistic field theories.

We have found the energy loss of the moving heavy point particle. For the zero temperature background, we found that particle loses energy, even at the zero temperature. Also, we considered the string theory dual to Lifshitz-like fixed points with anisotropic scale invariance, as proposed in [38], and studied the drag force. We found a non-zero drag force in the case of the zero temperature too. In this case, there are anisotropic and isotropic directions. We have found a non-zero drag force when particle is moving in these directions in (3.8). Then, we compared our results with drag force in the case of field theory whose symmetry group is the Schrödinger group, as shown in [13]. In this reference, the energy loss of the particle computed in the case of non-relativistic gravity dual to field theory with the Schrödinger CFT symmetry. Also, they found that a moving particle loses energy at the zero temperature. Based on these studies, we can conclude that this could be a common property of non-relativistic field theories. Holographic description of strongly correlated systems in condensed matter physics implies a non-zero drag force on a moving heavy carrier at the zero and finite temperature.

The most useful application of drag calculations in Lifshitz background has been described in [9]. They study the phenomenology of 'strange metals' and compute the electrical conductivity. It would be interesting to relate our results to the calculation of the DC conductivity.

In order to calculate the DC conductivity, an electric field should be activated on the D-brane probe and the resultant current $J^{x}$ can be computed in the boundary [10]. The conductivity $\sigma(E, T)$ is found from the Ohm's law

$\sigma(E, T)=\sqrt{\sigma_{0}^{2}+\sigma^{2}}$,

where $\sigma_{0}$ is a constant term arising from thermally produced pairs of the charge carriers. By increasing the mass of carriers, $\sigma_{0}$ can be made arbitrarily small and the leading term in conductivity will be $\sigma$. Based on the results of [10], we discuss the calculation of the leading term in conductivity, $\sigma$, by studying the properties of a moving single string. The authors in [10] found that

$\sigma^{2}=\left(\frac{2 \pi \alpha^{\prime}}{L^{2}}\right)^{2} r_{*}^{4}\left(J^{t}\right)^{2}$,

where $r *$ is the root of the following equation

$r^{2 z+2} f(r)-\left(2 \pi \alpha^{\prime}\right)^{2} E^{2}=0$.

An important result of [9] is based on the relation (48). This equation exhibits the power-law for the DC resistivity, $\rho \sim T^{2 / z} / J^{t}$. As discussed in ref. [9], this behavior is generic in a regime of dilute charge carriers coupled to a Lifshitz matter. Now, we derive this result from our drag force calculations.

We consider the quasi-particle description and write the 
equation of motion for them at the equilibrium, where the external force is $f=E$. At large mass limit, only charge carriers contribute to the current and one may express $J^{x}$ in terms of the velocity of the quasi-particles, $J^{x}=J^{t} v$. Regarding the Ohm's law ( $J^{x}=\sigma E$ ), one finds the leading term in conductivity as

$$
\sigma=\frac{v J^{t}}{E} \text {. }
$$

Based on the drag force calculations at the finite temperature and from the numerator of (16), one finds the velocity of the quasi-particle as

$$
v^{2}=r_{*}^{2 z-2} f\left(r_{*}\right)
$$

The drag force is related to the constant of the motion $C$, which can be found from the denominator of (16). Also, at equilibrium, $C^{2}=\left(2 \pi \alpha^{\prime}\right)^{2} E^{2}$; then

$$
E^{2}=\frac{L^{4} r_{*}^{2 z+2} f\left(r_{*}\right)}{\left(2 \pi \alpha^{\prime}\right)^{2}}
$$

From (51) and (52), one derives conductivity as

$$
\sigma=\frac{2 \pi \alpha}{L^{2}} r_{*}^{-2} J^{t}
$$

which is the same as (48). When the external field is very weak, one concludes that $r_{*} \sim r_{+}$; as a result, $\sigma \sim J^{t} / T^{2 / Z}$ confirms the result of [9].

It would be interesting to study the $R^{2}$ corrections to the DC conductivity for asymptotically Lifshitz backgrounds. One should consider the calculation of the drag force from (25). It is

\section{References}

1. J M Maldacena, Adv. Theor. Math. Phys. 2 (1998) 231.

2. S S Gubser, I R Klebanov and A M Polyakov, Phys. Lett. B 428 (1998) 105.

3. E Witten, Adv. Theor. Math. Phys. 2 (1998) 253.

4. E Witten, Adv. Theor. Math. Phys. 2 (1998) 505.

5. S A Hartnoll, Class. Quant. Grav. 26 (2009) 224002.

6. D T Son, Phys. Rev. D 78 (2008) 046003, K Balasubramanian and J McGreevy, Phys. Rev. Lett. 101 (2008) 061601.

7. S Kachru, X Liu and M Mulligan, Phys. Rev. D 78 (2008) 106005.

8. S Sachdev, "Quantum Phase Transitions," Cambridge University Press (1999).

9. S A Hartnoll, J Polchinski, E Silverstein and D Tong, "Towards Strange Metallic Holography," Journal of High Energy Physics 1004 (2010) 120.

10. A Karch and A O'Bannon, Journal of High Energy Physics 0709 (2007) 024.

11. A Karch and E Katz, Journal of High Energy Physics 0206 (2002) 043

12. S Kobayashi, D Mateos, S Matsuura, R C Myers and R M Thom-son, Journal of High Energy Physics 2007 (2007).

13. A Akhavan, M Alishahiha, A Davody and A Vahedi, Journal of High Energy Physics 2009, 03 (2009) 053.

14. P Koroteev and A V Zayakin, arXiv:0909.2551.

15. U H Danielsson and L Thorlacius, Journal of High Energy Physics 070 (2009) 0903.

16. C P Herzog, A Karch, P Kovtun, C Kozcaz, and L G Yaffe, Journal of High Energy Physics 0607 (2006) 013.

17. S S Gubser, Phys. Rev. D 74 (2006) 126005.

18. C P Herzog, Journal of High Energy Physics 0609 (2006) 032 . straightforward to find $\sigma R^{2}=2 \pi \alpha^{\prime} / L^{2} r_{*}^{-2} J^{t}$ and for small electric field, one concludes that $r_{*} \sim r_{+}$where $r_{+}$is the radial horizon related to the temperature of the matter (24). As a result, $R^{2}$ corrections to conductivity are given by

$$
\sigma_{R^{2}} \sim\left(\frac{4 \pi}{z_{0}+3+2 \lambda_{G B}\left(z_{0}-1\right)}\right)^{-2 / z} T_{H}^{-2 / z} J^{t} .
$$

From this equation, one can study the effect of the GaussBonnet coupling constant $\lambda_{G B}$ on the DC conductivity and resistivity. In the case of $z_{0}=1$, one finds the result of [9].

Calculating the DC conductivity at the zero temperature is straightforward. One should find the velocity of the massive charge from the numerator of (11); based on the relation between velocity and conductivity, one finds

$\sigma_{T=0}=\frac{2 \pi \alpha^{\prime}}{L^{2}} r_{c}^{-2} J^{t}$.

where $r_{c}$ is not the same as $r_{*}$ in (53).

It is interesting to investigate the calculation of conductivity from drag force in the type IIB string theory, as studied in [38]. We consider the finite temperature matter and turn on an small electric field in the "x" and "w" directions. In these directions, one finds that $\sigma_{x} \sim 1 / T^{2} \mathrm{v}$ and $\sigma_{\omega} \sim 1 / T^{4 / 3}$. As expected, conductivity is different in these directions.

\section{Acknowledgment}

I would like to thank M. Alishahhiha for very useful discussions; I should also appreciate J. McGreevy for reading the manuscript and providing comments.

19. G Bertoldi, B A Burrington, and A Peet, Phys. Rev. D 80 (2009) 126003.

20. G Bertoldi, B A Burrington, and A W Peet, Phys. Rev. D 80 (2009).

21. R B Mann, Journal of High Energy Physics 075 (2009) 0906.

22. M Taylor, arXiv: 0812. 0530.

23. D W Pang, Commun. Theor. Phys. 62 (2014) 256.

24. D W Pang, Journal of High Energy Physics 031 (2009) 0910.

25. D W Pang, Journal of High Energy Physics 1001 (2010) 116.

26. E J Brynjolfsson, U H Danielsson, L Thorlacius and $\mathrm{T}$ Zingg, J. Phys. A 43 (2010) 065401.

27. K Balasubramanian and J McGreevy, Phys. Rev. D 80 (2009) 104039.

28. E Ayon-Beato, A Garbarz, G Giribet and M Hassaine, Phys. Rev. D 80 (2009) 104029.

29. R G Cai, Y Liu, and Y W Sun, Journal of High Energy Physics 0910 (2009) 080.

30. S J Sin, S S Xu and Y Zhou, Int. J. Mod. Phys. A 26 (2011) 4617.

31. Y S Myung, Y W Kim and Y J Park, Eu. Phys. J. C 70 (2010) 335 .

32. A Ghodsi and E Hatefi, Phys. Rev. D 81 (2010) 044016.

33. A Ghodsi, Int. J. Mo. Phys. A 26 (2011) 925.

34. K B Fadafan, "Journal of High Energy Physics 0812 (2008) 051.

35. J F Vazquez-Poritz, arXiv: 0803.2890.

36. W Li, T Nishioka and T Takayanagi, Journal of High Energy Physics 0910 (2009) 015.

37. M Fujita, W Li, S Ryu and T Takayanagi, Journal of High Energy Physics 066 (2009) 0906. 
38. T Azeyanagi, W Li, and T Takayanagi, Journal of High Energy Physics 084 (2009) 0906.
39. J Kluson, Phys. Rev. D 81 (2010) 106006. 